Research Article

\title{
Shear-Dependent Yield Stress of Iron Ore Fine Tailings in Two-Step Flocculation Process
}

\author{
Ying Yang $\left(\mathbb{D},{ }^{1}\right.$ Hongjiang Wang $\mathbb{D}^{1},{ }^{1}$ Bern Klein, ${ }^{2}$ and Aixiang $\mathrm{Wu}{ }^{1}$ \\ ${ }^{1}$ School of Civil and Resource Engineering, University of Science and Technology Beijing, Beijing 100083, China \\ ${ }^{2}$ Norman B. Keevil Institute of Mining Engineering, University of British Columbia, Vancouver V6T 1Z4, Canada \\ Correspondence should be addressed to Hongjiang Wang; wanghj1988@126.com
}

Received 12 October 2020; Revised 26 October 2020; Accepted 29 October 2020; Published 18 November 2020

Academic Editor: Erol Yilmaz

Copyright (c) 2020 Ying Yang et al. This is an open access article distributed under the Creative Commons Attribution License, which permits unrestricted use, distribution, and reproduction in any medium, provided the original work is properly cited.

Both shear and flocculation have a significant influence on the rheological behavior of tailings, especially the yield stress. In the two-step flocculation process, the above two kinds of actions exist at the same time, and they influence each other. In order to explore the change rule of the yield stress and its internal causes, a two-step flocculation process of the iron ore fine tailings with different shear conditions in the four different phases was designed. In the primary flocculation phase, tailing particles combined with the primary flocculant and formed a primary floc network structure. In the primary broken phase, shear destroyed the primary floc network structure and decreased the average floc size, so the shear-dependent yield stress, the floc strength factor, and the fractal dimension decreased. In the secondary flocculation phase, broken floc combined with the secondary flocculant and produced a more compact floc network structure which had a better shear resistance. Therefore, in the secondary broken phase, with the increase of shear, the decrease of yield stress, the floc strength factor, and fractal dimension were less obvious than that in the primary broken phase. In both two broken phases, the yield stress of the secondary flocculating slurry was always higher than that of the primary flocculating slurry, but with the increase of shear, the difference became smaller. The floc strength and fractal dimension also showed the same rule. The internal reasons for the stronger shear resistance of the secondary flocculating slurry were the increase of the number of binding sites, the electric neutralization between the two flocculants, and the steric hindrance effect of the flocculants.

\section{Introduction}

For mineral processing and tailings disposal, dual polymer systems have been a research focus due to its significant advantages over the use of single polymers [1-3]. A dual polymer system can rapidly improve the dehydration capacity and rheological properties of the slurry. In mineral processing, rheological parameters, especially yield stress, directly affect energy consumption [4], separation efficiency [5], and sedimentation stability [6]. For tailings disposal, the physical stability of tailings, backfill, and paste are also dependent on rheological properties [7]. Therefore, it is necessary to fully understand the variation of rheology and the internal mechanism in a dual polymer system.

The interaction between particles and aqueous phases is the reason for rheological properties of slurry, which is dependent on physical characteristics and chemical characteristics of the constituents, such as mineralogical content [8], particle volume fraction $\varphi[9]$, particle size distribution [10], and chemical additives [11]. It is also affected by the external effect, such as shear action [12]. Therefore, rheological properties are a complex function of physical and chemical properties and of processes that occur at the scale of the suspended particles. In order to further understand rheological properties and its mechanisms, many pieces of research focused on the variation of rheological behavior under different conditions, such as shear, particle size distribution, floc structure, and maximum particle packing fraction.

In terms of minerals slurry, shear has an essential impact both on dual polymer systems and its rheological properties. Shear rate and shear time are considered to be the most 
important factors. For dual polymer systems, some researchers have used the product of shear rate and shear time as the index of shear action because this product is closely related to the rate of orthokinetic flocculation, which has a direct impact on the flocculation effect $[13,14]$. For rheological properties, shear action affects the rheological properties by changing the particle size distribution and the interaction of particles and the aqueous phase. For relatively small shear rates, the fine particles are controlled by Brownian motion effects or colloidal forces, while coarse particles are generally faced with frictional or collisional contacts or hydrodynamic forces [15]. However, for relatively high shear rates, the colloidal fine fraction and coarse fraction are assumed to act independently and the coarse fraction mainly contributes to increased viscosity through hydrodynamic dissipation [16, 17].

Apart from shear action, the rheological properties are closely related to floc structure and floc strength [18-21]. Nasser and James [22] found that the magnitude of the yield stress and viscous modulus is strongly dependent upon the floc structure. When shear exceeds the particle interactions, the floc network structure is disrupted, and the pulp yield stress decreases, which shows clear links between shear, floc structure, and dewaterability [23]. Furthermore, Farrokhpay [24] proposed that the rheological behavior of mineral slurries indicates the level of particle interaction or aggregation and disaggregation is a balance between floc formation and floc breakage [25-29]. In other words, the shear-dependent yield stress of slurry indicates the shear resistance of the floc network structure in the process of flocculation and shear disruption. And in the field of water treatment, the floc strength factor demonstrates the shear resistance of flocs in the process of floc formation and floc breakage. Therefore, the floc strength factor can be considered as a unit of shear resistance of the floc network, and it can also be used as an important indicator of the variation of the shear-dependent yield stress. However, there is not enough information on the relationship between yield stress, floc structure, and floc strength factor in the two-step flocculation process.

In the paper, a two-step flocculation process of the iron ore fine tailings under different shear conditions was designed so as to explore the change rule of the yield stress with shear. This two-step flocculation process included the primary flocculation phase, the primary broken phase, the secondary flocculation phase, and the secondary broken phase. In order to explain the variation of the shear-dependent yield stress in this process and its internal causes, the floc size, floc strength factors, and the fractal dimension will be calculated and analyzed.

\section{Materials and Methods}

2.1. Tailings. The tailings used in this study were iron ore tailings originally supplied by ArcelorMittal USA, a steel and mining company operating in the USA. The tailings preparation process is comprised of a number of steps. Firstly, taking a $45 \mathrm{~kg}$ wet sample of $+4.0 \mathrm{~mm}$ feed size from the tailings and passing it through a vibrating screen. The portion of the tailings that did not pass through the screen was crushed using gyratory and cone crushers and subsequently mixed with the portion of the sample that had filtered through. Secondly, the combined sample was then mixed with water to bring its concentration to $60 \%$ prior to grounding it in a rod mill for 66 mins. Through preexperiments, the relation curve between the particle size distribution of tailings and grinding time was obtained and the grinding time was determined to be 66 mins with the 80th percentile of the cumulative particle size distribution of $70 \mu \mathrm{m}$ as the target. Then, the sample was subsequently dried in a drying box until the tailings were completely dry so as to obtain the working sample that would be used in this study. The working sample particle size distribution was measured using a Malvern Mastersizer 2000 Laser Diffraction Particle Size Analyser. The 50th and 80th percentile of the cumulative particle size distribution was $60.2 \mu \mathrm{m}$ and $70.2 \mu \mathrm{m}$, respectively (Figure 1). The specific gravity of the tailings was $2.326 \mathrm{~g} / \mathrm{cm}^{3}$, and the result of the X-ray diffraction is shown in Table 1.

2.2. Flocculants. Through single flocculation preexperiments and combined flocculation preexperiments, the optimal flocculant combination was selected (923VHM and $4800 \mathrm{SSH}$ ). These two kinds of commercial flocculants were used in this study, supplied by SNF Canada. The anionic polyacrylamide, 923VHM, was used as the primary flocculant and the cationic polyacrylamide, 4800SSH, was used as the second flocculant. Both of them have high molecular weight and good water solubility. To prevent mineral impurities from affecting test results, deionized water was used in all water-based tests.

2.3. Suspension Preparation. A slurry of $15 \%$ solids (w/v \%) was prepared in a $1 \mathrm{~L}$ beaker using $150 \mathrm{~g}$ tailings and $850 \mathrm{ml}$ deionized water. The slurry was homogenized by agitating at a rotational speed of approx. $600 \mathrm{rpm}$ for 30 seconds, and at $280 \mathrm{rpm}$ for $10 \mathrm{mins}$. Then $2 \mathrm{ml}$ of the anionic PAM flocculant solution with the concentration of $0.15 \%$ was added to the slurry (corresponding to the polymer optimum dosage of $20 \mathrm{~g} / \mathrm{t}$, determined from previous experimentation) and agitation was ceased immediately to avoid breaking the flocs. Following agitation, the beaker containing the slurry was allowed to rest for 5 mins prior to removing the supernatant from the solution by pouring (it out of the beaker). The average particle size, $d_{0}$, of the underflow was measured by using the Malvern Mastersizer 2000 Laser Diffraction Particle Size Analyser.

2.4. Rheology Tests in Two-step Flocculation Process. The time-dependent rheological behavior of the slurry was characterized using a shear rheometer (Thermo Haake VT 550, Germany). The rheometer was also used as a power device to provide floc shear disruption during the two-step flocculation process. The cup and bob fixture was a concentric cylinder geometry of which the radius of the fixed outer cylinder and the rotatable inner cylinder was $21.00 \mathrm{~mm}$ 


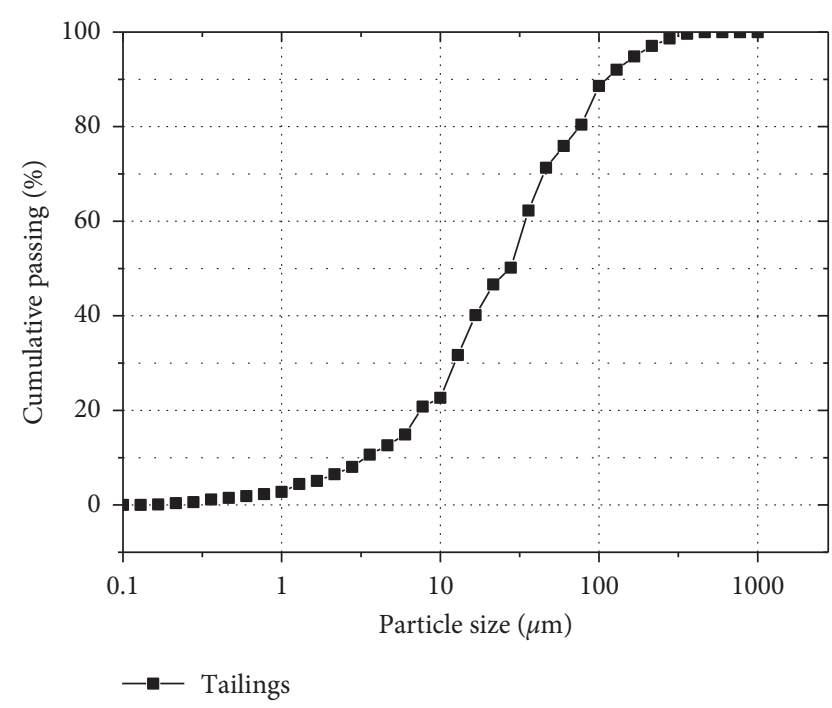

Figure 1: Granularity composition of tailings.

TABle 1: Parameter design of floc shear disruption.

\begin{tabular}{lccc}
\hline \multicolumn{2}{c}{ Primary broken phase } & \multicolumn{2}{c}{ Secondary broken phase } \\
Shear rate & Shear time & Shear rate & Shear time \\
\hline 200 & 30 & 200 & 30 \\
400 & 30 & 400 & 30 \\
600 & 30 & 600 & 30 \\
800 & 30 & 800 & 30 \\
200 & 60 & 200 & 60 \\
400 & 60 & 400 & 60 \\
600 & 60 & 600 & 60 \\
800 & 60 & 800 & 60 \\
200 & 120 & 200 & 120 \\
400 & 120 & 400 & 120 \\
600 & 120 & 600 & 120 \\
800 & 120 & 800 & 120 \\
200 & 240 & 200 & 240 \\
400 & 240 & 400 & 240 \\
600 & 240 & 600 & 240 \\
800 & 240 & 800 & 240 \\
\hline
\end{tabular}

and $20.04 \mathrm{~mm}$, respectively, so there was a gap between the cup and bob fixture to minimize non-Newtonian shear rate effects, particle bridging across the shear surfaces, and the magnitude of wall slip errors [30-32]. For each test, $34 \mathrm{ml}$ samples were required and the temperature was fixed at $23^{\circ} \mathrm{C}$ by the Poly science 9100 constant temperature circulator from the USA.

The rheological tests were conducted including four phases, primary flocculation phase, primary broken phase, secondary flocculation phase, and secondary broken phase, as shown in Figure 2.

Firstly, in the primary flocculation phase, the tailings slurry and the primary flocculant solution were mixed as shown previously, to prepare the primary flocculated suspension. The yield stress of the primary flocculated suspension was measured with the increase of shear rate from 0 to $300 s^{-1}$ and the decrease from $300 s^{-1}$ to 0 within 120 seconds. In addition, the primary particle size distribution, $d_{1}$, was obtained using the Malvern Mastersizer 2000 Laser Diffraction Particle Size Analyser.

Secondly, in the primary broken phase, the tests were implemented at a constant shear rate for a desired period (Table 1), during which the rheometer was only used as a floc shear disruption device. Subsequently, the yield stress and the particle size distribution, $d_{2}$, of the primary broken suspension was measured with the same method in the primary flocculation phase.

Then, in the secondary flocculation phase, $0.4 \mathrm{ml}$ flocculant solution of the cationic PAM with the concentration of $0.15 \%$ (corresponding to the polymer optimum dosage of $20 \mathrm{~g} / \mathrm{t}$, determined from previous experimentation) was added to the suspension in the cup and the slurry was stirred until it was homogeneous. Then, the measurement of the yield stress and the particle size distribution, $d_{3}$, of the secondary flocculated slurry was carried out.

Finally, in the secondary broken phase, adjusting the shear rate and shear time to the same values with those in the primary broken phase (Table 1), prior to measuring the yield stress and the particle size, $d_{4}$, of the secondary broken suspension.

2.5. Floc Structure Measurement. At the end of each phase of the experiments, samples were taken from the main suspensions. The microstructure of flocs was observed by SEM. In order to make the images obtained clearly reflect the changes of the floc microstructure, with the help of ImageJ2X software, the images were binarized by gray histogram method.

\subsection{Data Treatment Methods}

2.6.1. Floc Strength Factor in Two-Step Flocculation. Floc strength factor demonstrates the shear resistance of flocs in the process of floc formation and floc breakage, while sheardependent yield stress of slurry indicates the shear resistance of the floc network in the process of flocculation and shear disruption. Therefore, floc strength factor can be considered as a unit of shear resistance of the floc network and it can also be used as an important indicator of the variation of the shear-dependent yield stress.

In the paper, the floc strength factor was introduced from the water treatment field to explore the relationship between floc strength factor and shear-dependent yield stress of the slurry in the two-step flocculation process. The formulas were listed as follows [33]:

Strength factor:

$$
F_{1}=\frac{d_{2}}{d_{1}} .
$$

Here $d_{1}$ is the average flocs size before breakage, $\mu \mathrm{m}, d_{2}$ is the average flocs size after breakage, $\mu \mathrm{m}$. 


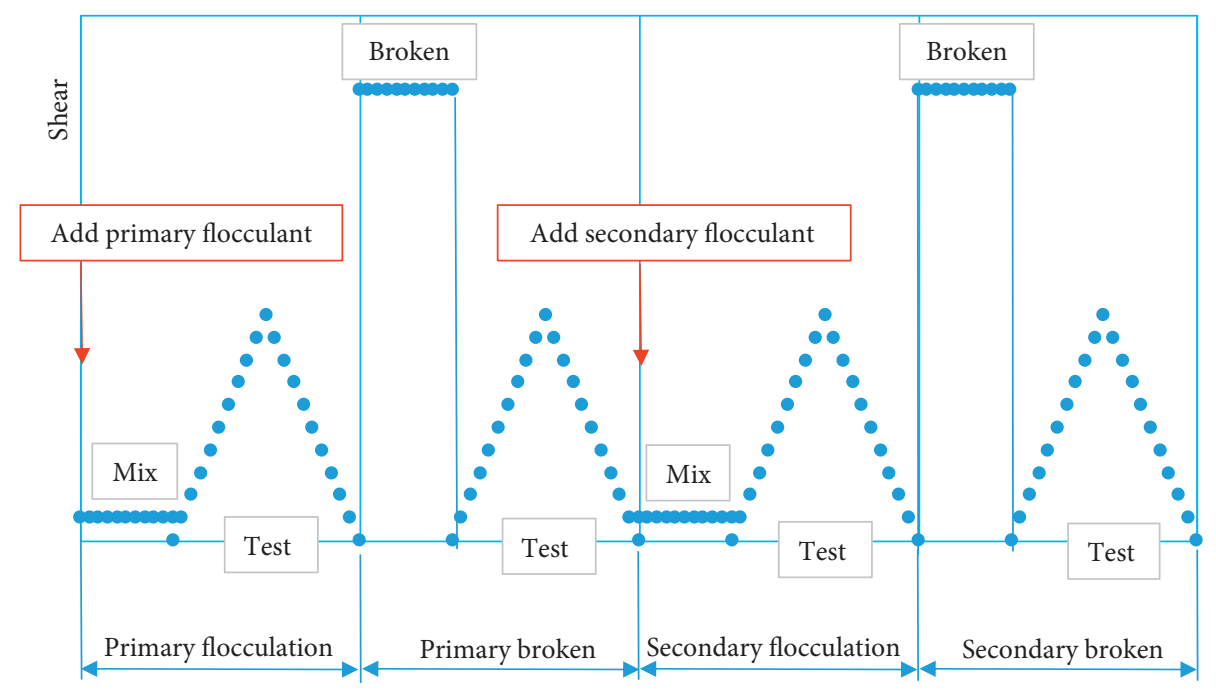

Figure 2: A scheme of rheological tests.

2.6.2. Floc Fractal Dimension in Two-Step Flocculation. Floc fractal dimension is an important parameter of floc structure, floc strength, and flocculation effect. It can also be used as an important indicator to explain the variation of the shear-dependent yield stress in the two-step flocculation.

With the help of FractalFox image fractal processing software, the box counting dimension method was used to calculate the fractal dimension of floc structure in the twostep flocculation experiments. The basic principle was as follows. The small box with side length $r$ was used to cover the fractal curve, and the number of small boxes covering the fractal curve was $N(r)$. Then, the box side length $r$ was continuously reduced. When $r$ infinitely approached zero, the fractal dimension $D$ was obtained.

$$
D=-\lim _{r \longrightarrow 0} \frac{\log N(r)}{\log r} .
$$

\section{Results}

3.1. Yield Stress in the Primary and Secondary Broken Phases. The Bingham model was used to fit the measurement results of the rheometer to obtain the yield stress of the slurry in the four different phases. They were the first phase (primary flocculation phase), the second phase (primary broken phase), the third phase (secondary flocculation phase), and the fourth phase (secondary broken phase), and the calculated coefficient of determination $R^{2}$ was $>0.95$, which shows that the Bingham model can accurately reflect the change of the rheological properties in different phases.

The variation of yield stress in the primary flocculation and primary broken phases with a shear rate under different shearing time is shown in Figure 3. In the primary broken phase, the yield stress gradually decreased with the increase of shear rate and shear time and the minimum value was about $80 \%$ of the yield stress without shear disruption. The increase of shear rate led to a more significant decrease in yield stress than that of shear time. The failure of the floc network structure was a possible reason for this phenomenon. The increasing shear action aggravated the disruption of the floc network structure, resulting in the continuous reduction of the yield stress in the broken phase. In addition, the increasing shear rate could overcome the stronger internal effect of floc structure and change the failure mode of floc structure, while the increase of shear time could enlarge the damage degree of floc structure.

As shown in Figures $4(\mathrm{a})-4(\mathrm{~d})$, in the primary and secondary broken phases, the yield stress obviously decreased with the increase of shear rate and shear time. However, the yield stress in the secondary broken phase decreased more slowly than that in the primary broken phase. Taking Figure 4(d) as an example, when the shear time was $240 \mathrm{~s}$, with the increase of shear rate from $200 \mathrm{~s}^{-1}$ to $400 \mathrm{~s}^{-1}$, the yield stress in the primary broken phase decreased by $3.5 \mathrm{~Pa}$, while that in the secondary broken phase decreased by $1.5 \mathrm{~Pa}$.

In addition, the yield stress in the secondary broken phase was always larger than that in the primary broken phase and the gap became smaller with the increase of shear rate and shear time. In other words, secondary flocculation increased the yield stress and shear resistance of the slurry. This could be explained by the increase of the floc strength and the density of floc structure in the secondary flocculation process, which will be further analyzed in Sections 3.3 and 3.4. Furthermore, with the increase of shear action, the smaller gap of the yield stress between the primary broken phase and the secondary broken phase indicated that the stronger shear resistance of the secondary flocculation slurry was more obvious under low shear action and it was weakened under high shear action.

For dual polymer systems, the product of shear rate and shear time is regarded as the index of shear action because it is closely related to the rate of orthokinetic flocculation, which has a direct impact on the flocculation effect $[13,14]$. Therefore, in this paper, the product of shear rate and shear time was used as a shear index to explore the variation of yield stress in the primary broken and secondary broken phases. 


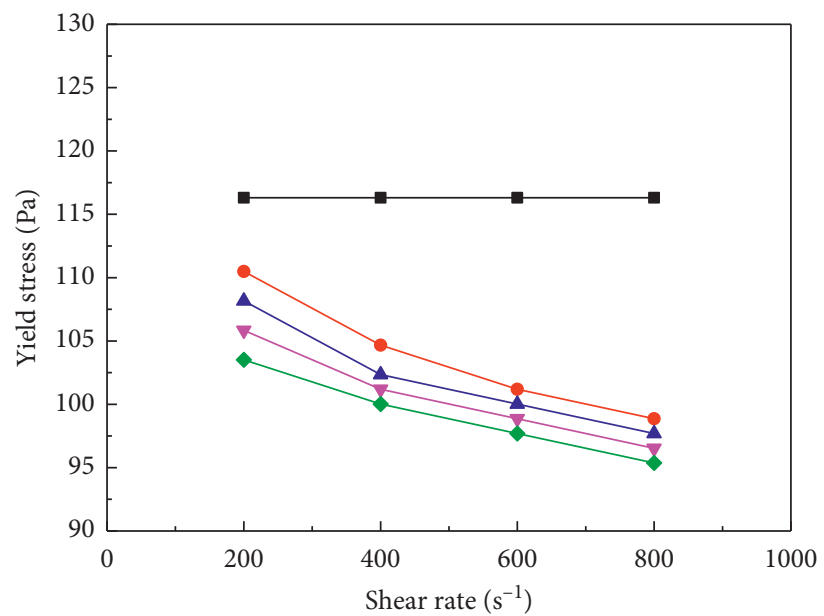

- Primary flocculation phase

- Primary broken phase $(t=30 \mathrm{~s})$

$\triangle$ Primary broken phase $(t=60 \mathrm{~s})$

$\rightarrow$ Primary broken phase $(t=120 \mathrm{~s})$

$\rightarrow$ Primary broken phase $(t=240 \mathrm{~s})$

FIGURE 3: The variation of yield stress with shear rate during different shear times in the primary flocculation and primary broken phases.

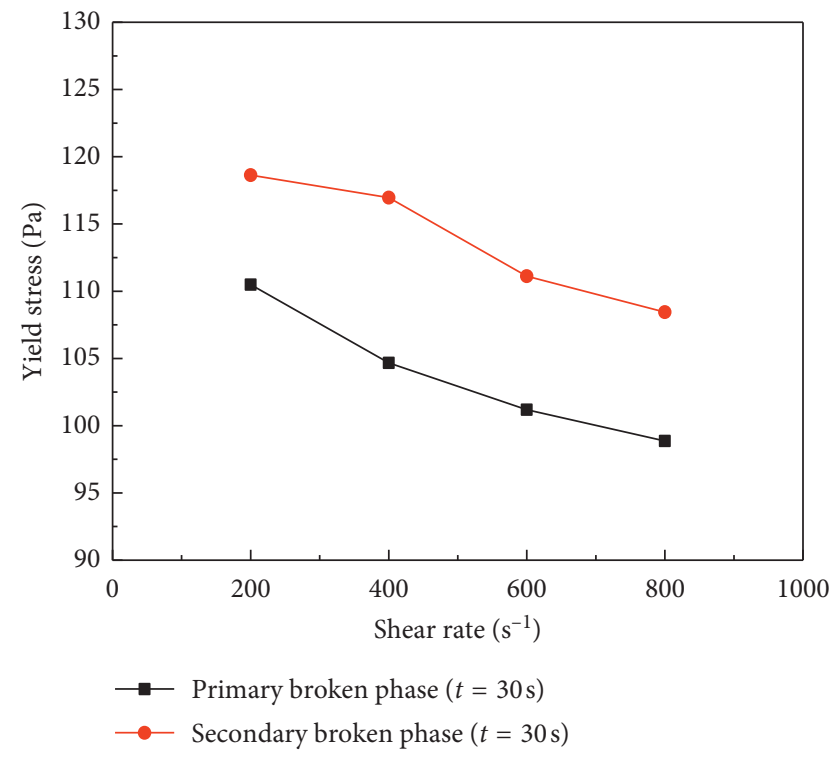

(a)

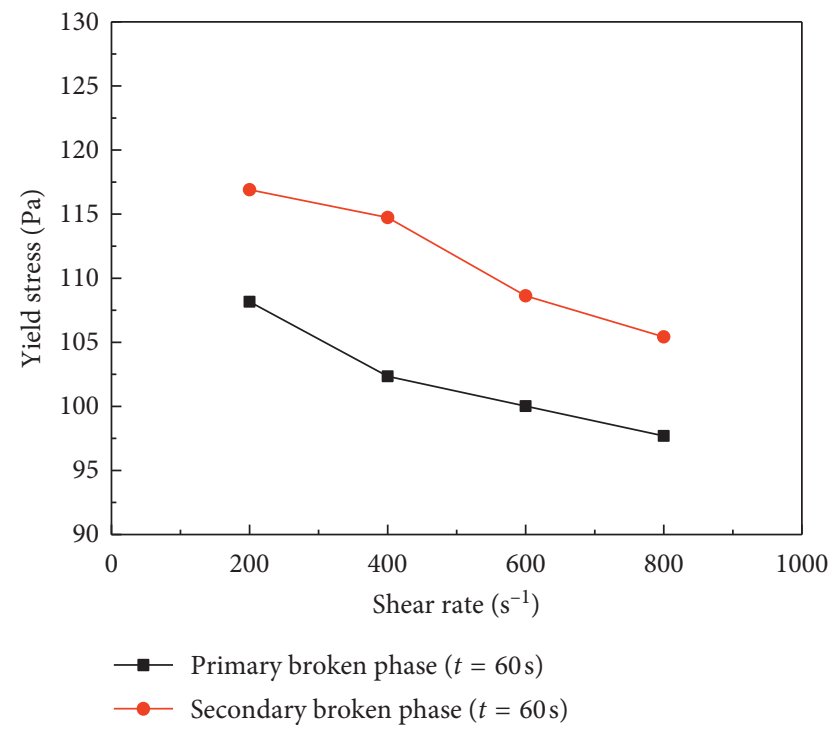

(b)

Figure 4: Continued. 


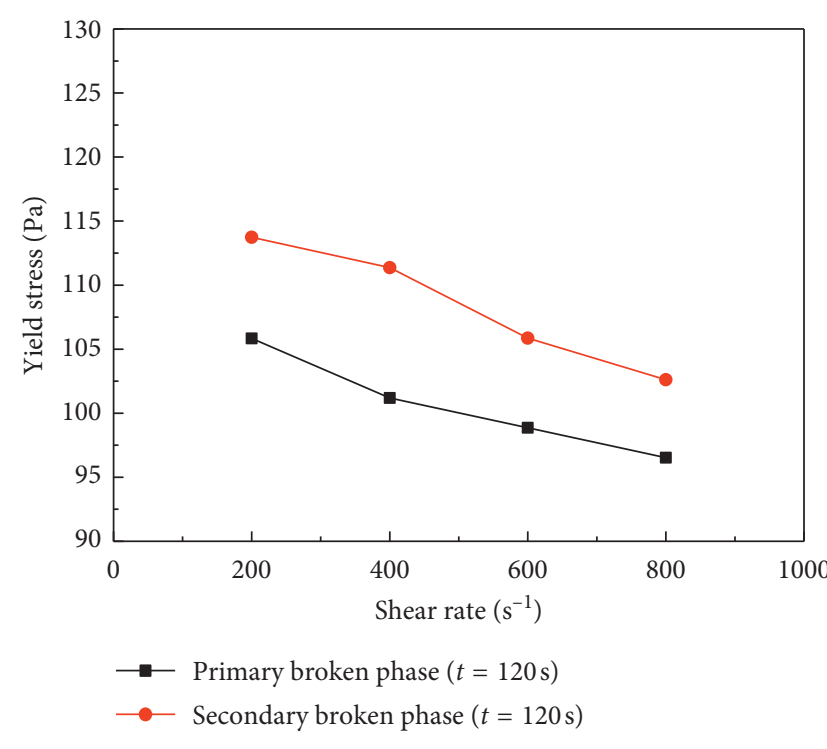

(c)

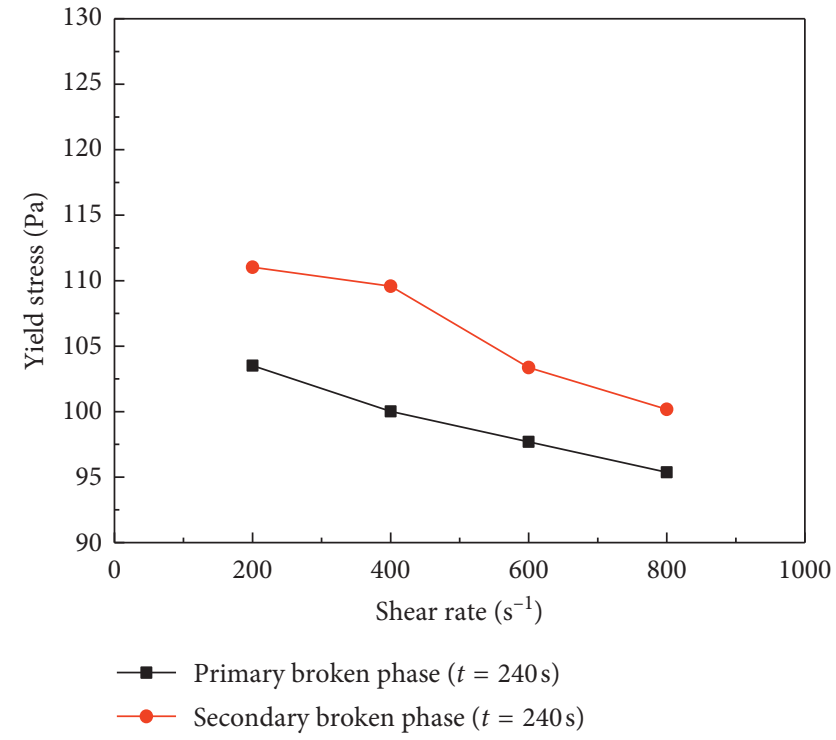

(d)

Figure 4: The variation of yield stress with shear rate during different shear times in the primary broken and secondary broken phases.

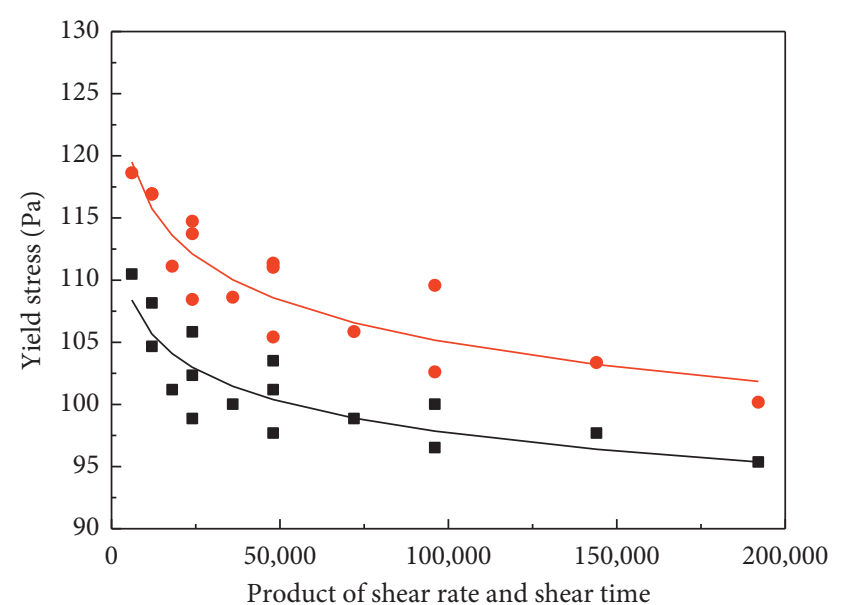

- Primary broken data

- Secondary broken data

- Fitting curve of primary broken phase

_ Fitting curve of secondary broken phase

FIgURE 5: The variation of yield stress with the product of shear rate and shear time in the primary and secondary flocculation phases.

As shown in Figure 5, the yield stress in the primary and secondary broken phases showed a similar change trend, but the value in the secondary broken phase was always higher than that in the primary broken phase. When the product of shear rate and shear time was between 0 and 100,000, the yield stress in the primary broken and secondary broken phases decreased significantly. When the value was higher than 100,000, the yield stress in the two broken phases tended to be stable. The probable reason for this phenomenon was the shear was easier to overcome the weak connections of floc structure, and it had a more obvious effect on the yield stress of slurry. The higher yield stress in the secondary broken phase was probably because secondary flocculation formed a more compact floc network structure and higher floc strength.

3.2. Floc Size in the Two-Step Flocculation. The variation of average floc size in the primary flocculation and primary broken phases with a shear rate under different shearing time is shown in Figure 6. In the primary broken phase, the average floc size gradually decreased with the increase of shear rate and shear time, and the minimum value was about $80 \%$ of the average floc size without shear disruption in the primary flocculation phase. The increase of shear rate led to a more significant decrease in yield stress than that of shear time.

The possible reason for this phenomenon was that the complete floc network structure formed in the primary flocculation phase was too loose. There were some weak connections in the primary floc network structure, so the shear resistance of the whole structure was low. In the primary broken phase, even in the face of low shear, the structural integrity was significantly damaged, which led to the average floc size decreasing rapidly.

As shown in Figure 7, compared with the primary floc, the average size of secondary floc increased significantly. In the secondary broken phase, with the increase of shear rate and shear time, the average size of secondary floc also decreased gradually, but the size of secondary broken floc after shear was still larger than that of primarily broken floc. The minimum size of the secondary broken floc was $83 \%$ of the secondary floc without shear failure. In other words, secondary flocs had higher floc strength. This may be because in the primary broken phase, the shear action destroyed the weak connection parts in the loose primary floc network structure, and the internal effect of the remaining flocs was strong. At the same time, the secondary flocculation 


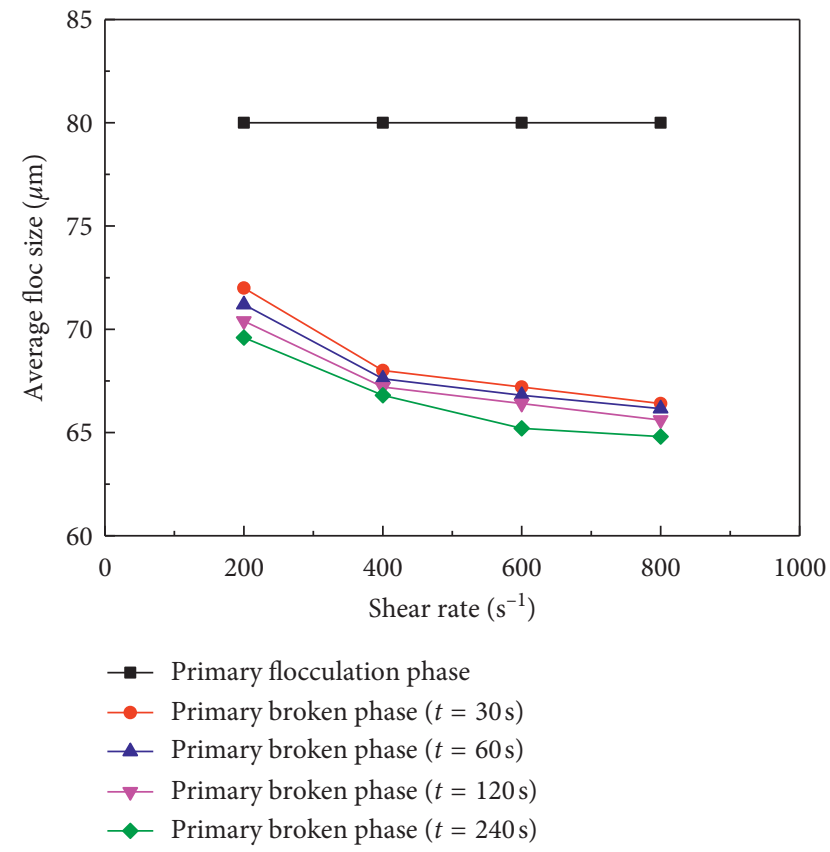

FIGURE 6: The variation of average floc size with shear rate during different shear times in the primary flocculation and primary broken phases.

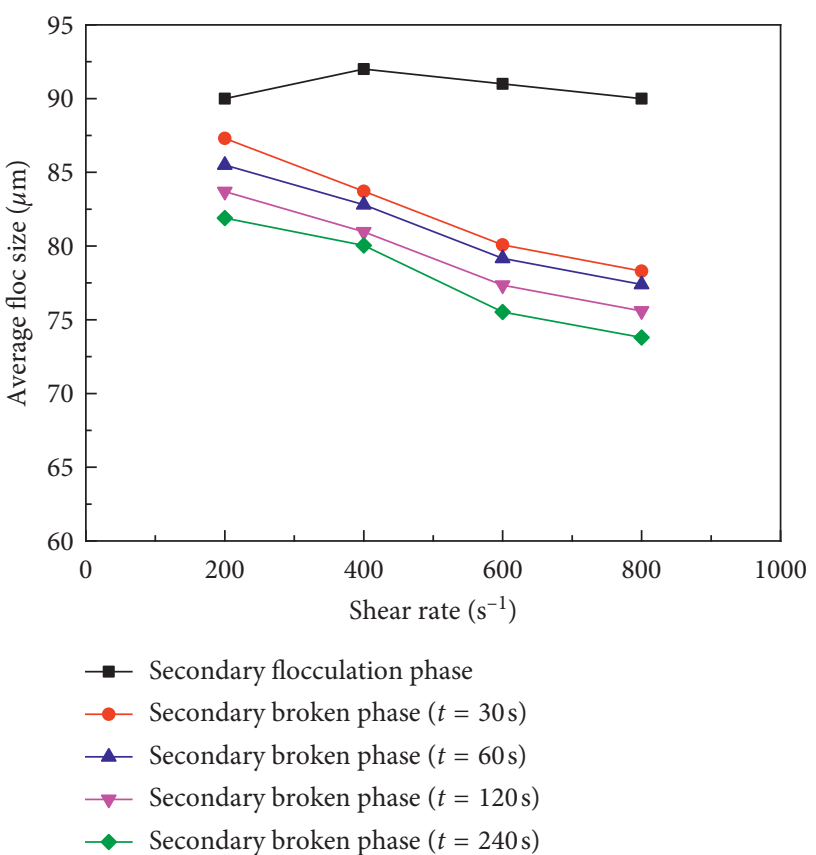

FIgURE 7: The variation of average floc size with shear rate during different shear times in the secondary flocculation and secondary broken phases.

produced a dense structure, which not only increased the shear resistance of the whole structure but also increased the strength of the internal flocs.

As shown in Figure 8, with the increase of the product of shear rate and shear time, the change trend of the average floc size in the primary and secondary broken phases was

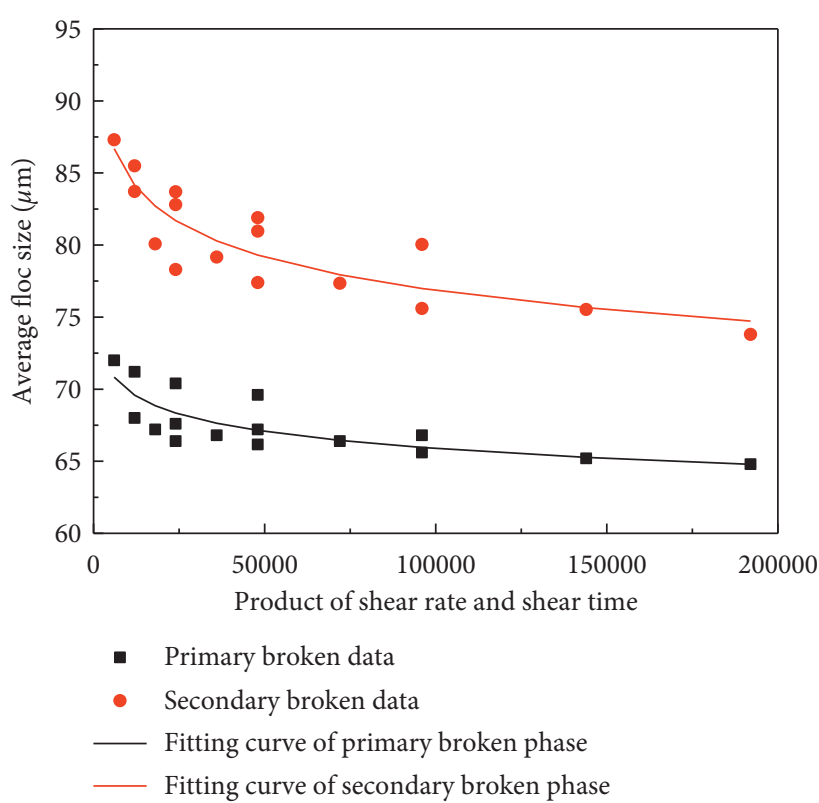

FIGURE 8: The variation of average floc size with a product of shear rate and shear time in the primary and secondary broken phases.

consistent with that of yield stress. The average floc size in the two broken phases decreased rapidly and then tended to be stable when the product of shear rate and shear time was higher than 100,000. The average floc size decreased rapidly probably because lower shear overcame weaker connections of floc structure, which caused a large-scale structural fracture of floc structure, the integrity of the floc network structure was significantly damaged. In addition, the average floc size in the secondary broken phase was also always higher than that in the primary broken phase. It means that the strength of the secondary floc was higher than that of the primary floc.

\subsection{Floc Strength Factor in the Primary and Secondary Broken} Phases. According to equation (1), the floc strength factors were calculated using the results of the average floc sizes. As shown in Figure 9, the secondary floc strength factors were always larger than the primary ones and the gap became smaller with the increase of shear rate and shear time. In other words, both the shear disruption prior to the secondary flocculation and secondary flocculation process enhanced the floc shear resistance and formed the stronger secondary flocs.

With the increase of shear disruption, the primary and secondary floc strength factors decreased gradually and finally tended to be stable. The variation of the floc strength factors could be explained by analyzing the change of the average floc sizes. As shown in Figures 6 and 7, the difference between the average sizes of the flocs and the broken flocs in the primary flocculation process was always larger than that in the secondary flocculation process. It led to the secondary floc strength factors being larger than the primary ones. In addition, secondary flocculation enhanced the floc sizes and further widened the difference 


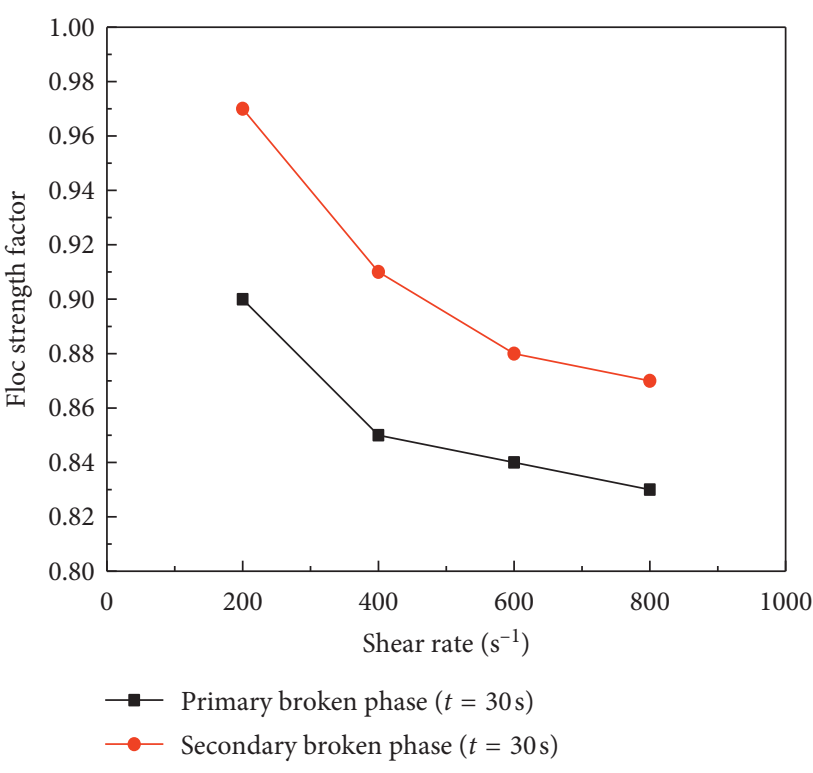

(a)

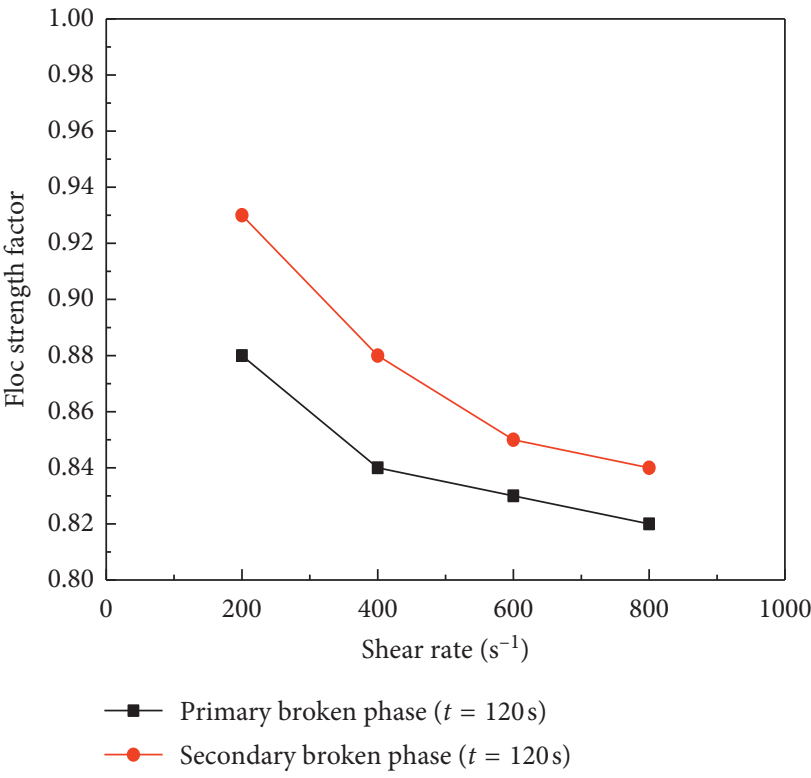

(c)

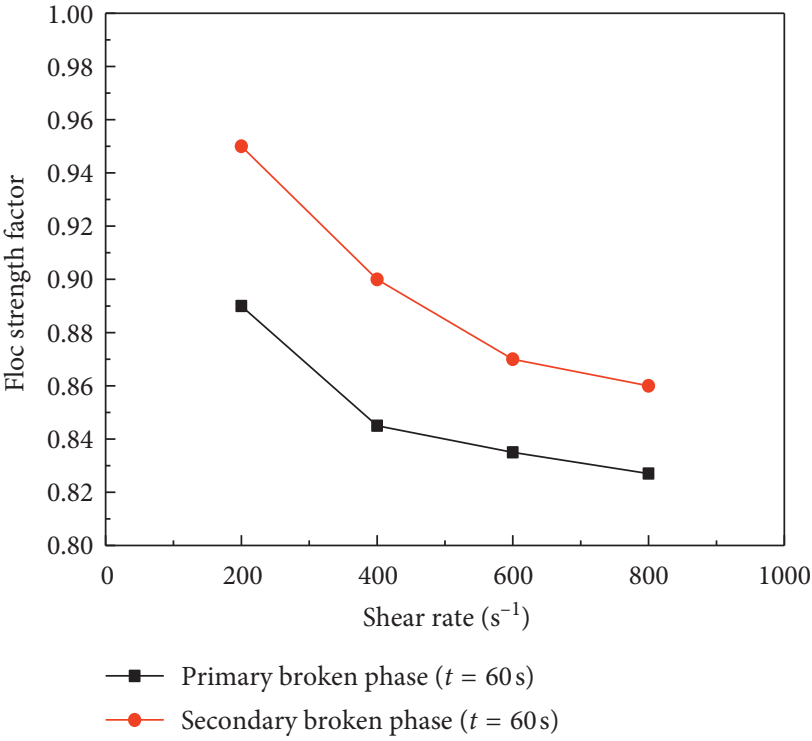

(b)

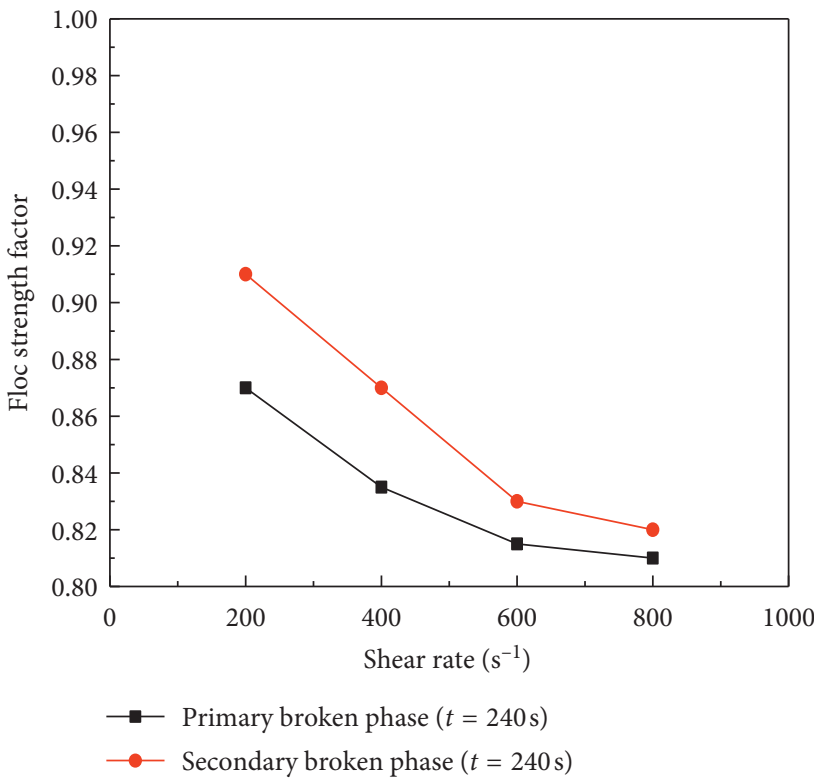

(d)

FIGURE 9: The variation of floc strength factor with shear rate during different shear times in the primary broken and secondary broken phases.

between the floc strength factors in the primary and secondary broken phases.

The variation of floc strength factors with the product of shear rate and shear time in the primary and secondary broken phases is shown in Figure 10. When the product of shear rate and shear time was between 0 and 100,000, the floc strength factors decreased significantly. When the value was higher than 100,000, the floc strength factors tended to be stable. The secondary floc strength factors were always larger than the primary ones, and the gap became smaller under higher shear. The variation of floc strength could also be explained by the failure mode of floc structure under shear and the strengthening floc in the secondary flocculation.
It is worth mentioning that the variation of yield stress, floc size, and floc strength with the product of shear rate and shear time in the primary and secondary broken phases being basically consistent (Figures 5, 8, and 10). Their inflection points from a rapid decrease to stabilization were all around the product value of 100,000 . This could be explained by the increase of the strength of floc or floc structure in the secondary flocculation process. The strengthening effect was more obvious under lower shear stress. According to the definition of floc strength, the change of floc size was the reason for the change of floc strength. In other words, secondary flocculation increased the yield stress of the slurry by enhancing the floc strength. However, the change of the floc strength was not the only reason for the variation of the 


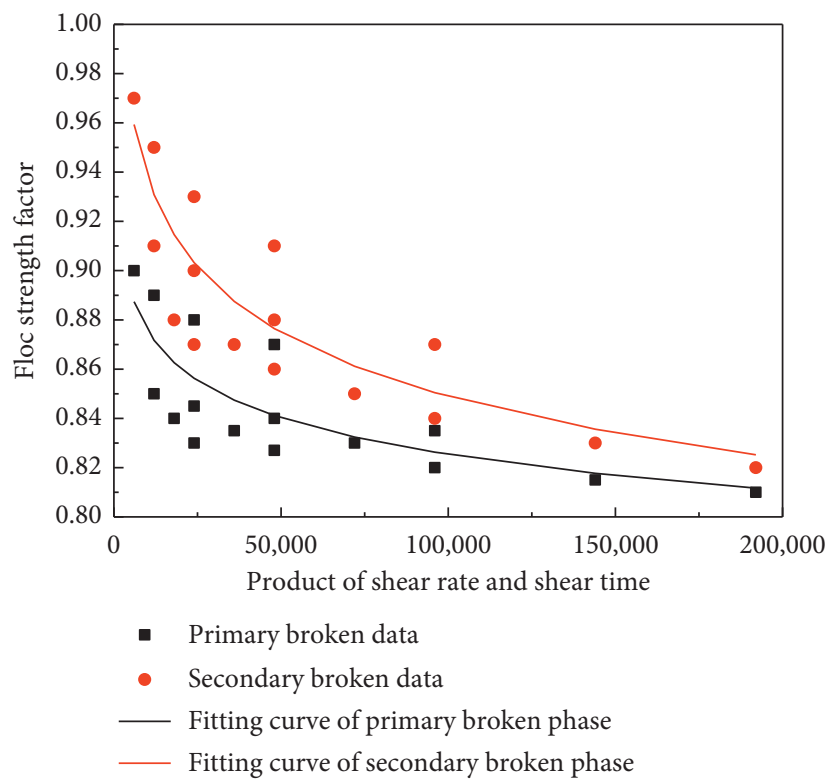

FIGURE 10: The variation of floc strength factor with the product of shear rate and shear time in the primary and secondary broken phases.

yield stress. It was also related to the floc network structure, which will be explained in Section 3.4.

\subsection{Floc Fractal Dimension in the Two-Step Flocculation.} With the help of the box counting dimension method, the fractal dimensions of floc structure were calculated in the two-step flocculation experiments. The curve of the shearing time of $120 \mathrm{~s}$ was taken as an example. The variation of fractal dimensions of floc structure with the increase of shear rate and shear time was shown in Figure 11. Compared with the primary and secondary flocculation, the secondary floc fractal dimensions were all higher than the primary ones. Similarly, the fractal dimensions of the secondary broken floc were also higher than those of the primary broken ones. In addition, under higher shear, the difference between the fractal dimension in the primary (broken) and secondary (broken) phases became smaller.

As shown in Figure 12, the floc structure in different phases was significantly different. In the primary flocculation phase, there were many "cavities" in the floc network structure, which led to loose floc structure, low shear strength, and small fractal dimension. In the primary broken phase, shear destroyed the floc structure and produced a large number of small flocs. In the secondary flocculation phase, the floc size increased significantly, the structure became more compact, which led to high floc strength and large fractal dimensions. In the secondary broken phase, under the same shear action, the scale of broken floc structure was smaller and the sizes of broken flocs were larger compared with the primary broken phase.

As shown in Figure 13, in the primary and secondary broken phases, the change trend of fractal dimension of floc structure was consistent with that of yield stress with the increase of shear rate and shear time. In other words, the variation of the floc fractal dimension could also be another reason for the change of yield stress in the two-step

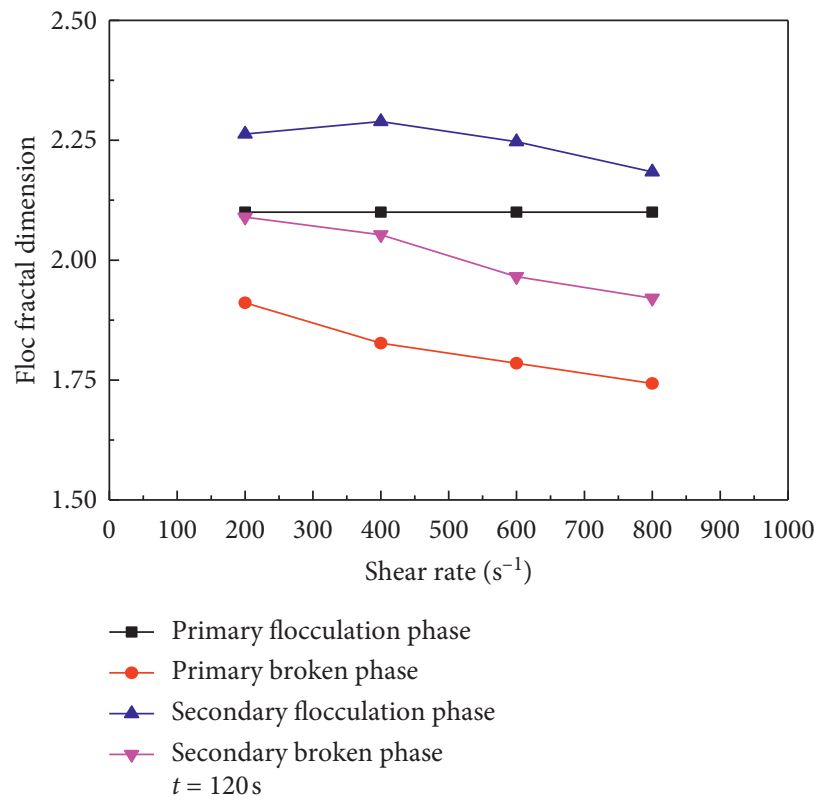

FIgURE 11: The variation of floc fractal dimension with a shear rate in the four phases (shear time $=120 \mathrm{~s}$ ).

flocculation. Therefore, secondary flocculation increased the yield stress of the slurry by enhancing the floc strength and the density of the floc structure.

3.5. Discussion. Through analyzing the variation of yield stress, floc size, floc strength, and fractal structure with the increase of shear rate and shear time, comparing the change rule of the above parameters in the primary broken phase with that in the secondary broken phase, it is found that the secondary flocculation slurry had better shear resistance. This may be because of the following (Figure 14): 


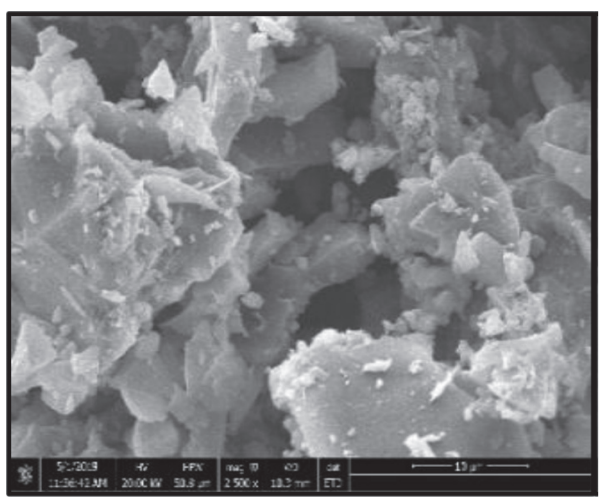

(a)

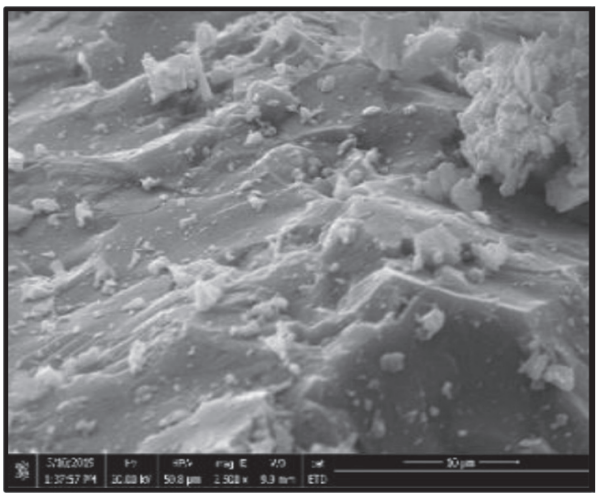

(c)

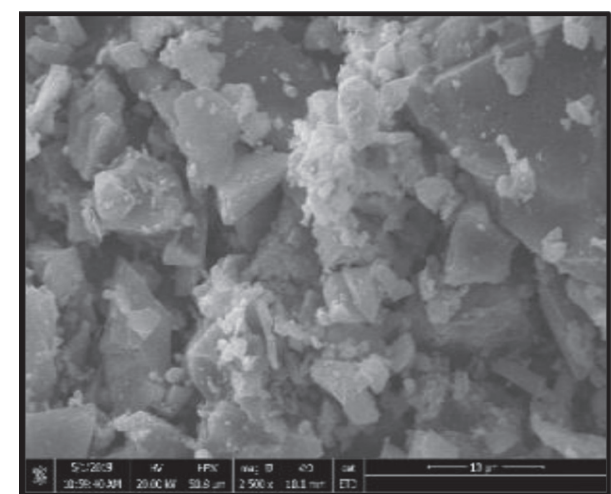

(b)

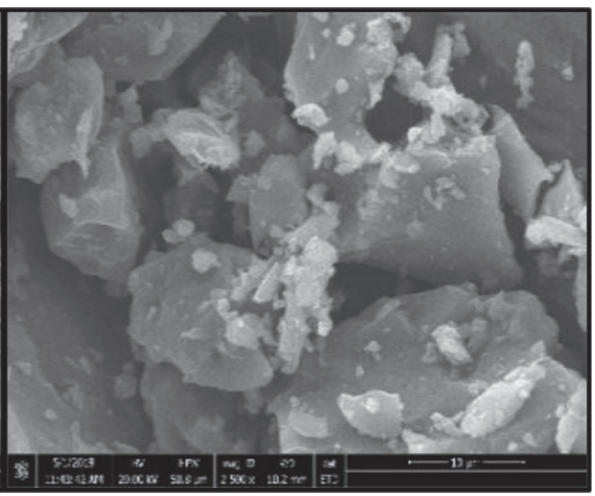

(d)

FIGURE 12: The SEM of floc structure in the four phases (shear rate $=400 \mathrm{~s}^{-1}$ and shear time $=120 \mathrm{~s}$ ): (a) primary flocculation, (b) primary broken, (c) secondary flocculation, and (d) secondary broken.

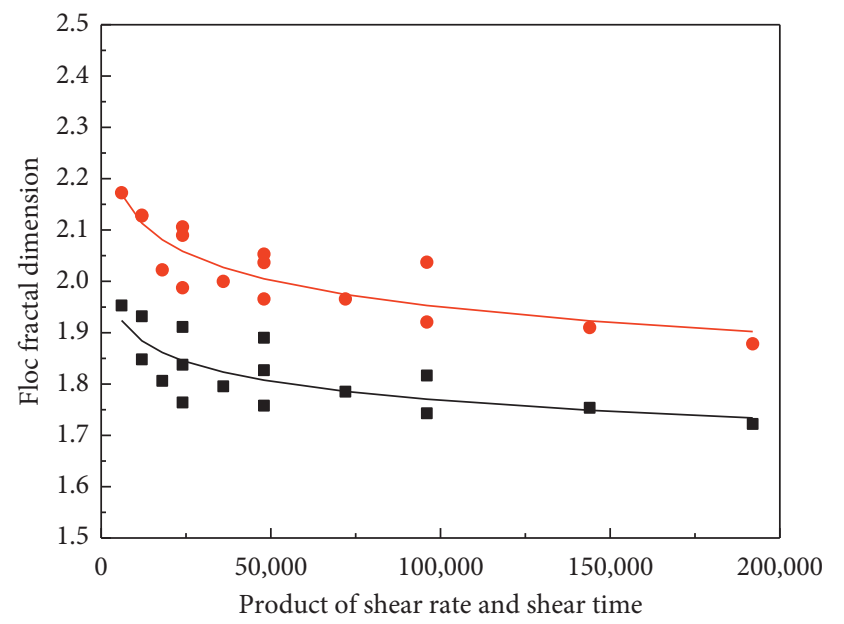

- Primary broken data

- Secondary broken data

__ Fitting curve of primary broken phase

__ Fitting curve of secondary broken phase

FIGURE 13: The variation of floc fractal dimension with the product of shear rate and shear time in the primary and secondary broken phases.

(1) The addition of primary flocculant increased the number of binding sites on the surface of tailings particles; in the primary broken phase, shear

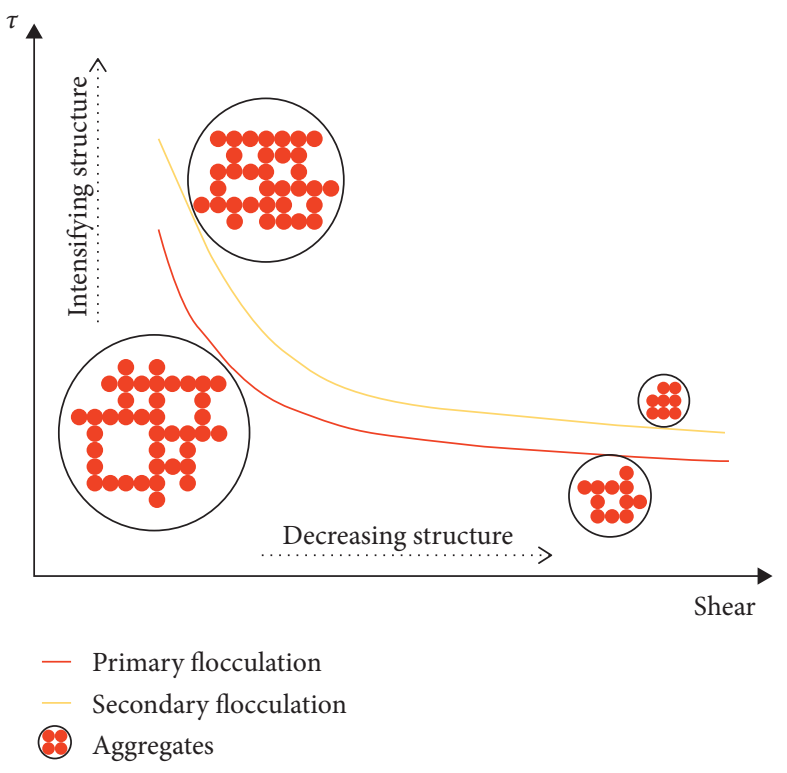

FIGURE 14: A schematic for the variation of shear-dependent yield stress with shear in the two-step flocculation process.

disruption promoted the release of binding sites on the surface of broken flocs. Therefore, in the secondary flocculation phase, the binding probability of 
broken flocs and secondary flocculant molecules increased.

(2) In this experiment, the anionic flocculant was used as the primary flocculant, and the cationic flocculant was used as the secondary flocculant. Through the electric neutralization of the two kinds of flocculants, secondary flocculation formed a more compact floc network structure. Compared with the primary floc network structure formed by bridging, the internal force of the secondary floc network structure was stronger which showed stronger shear resistance.

(3) The steric hindrance effect of the two polymeric flocculants gave them higher bridging probability, which was conducive to the formation of dense floc network structure.

\section{Conclusions}

The variation of the yield stress, the floc strength factor, and the fractal dimension of the iron ore fine tailings under different shear conditions in a two-step flocculation process was studied in the research through analyzing the change of floc network structure and floc size distribution. The main conclusions are as follows:

(1) Shear destroyed the floc network structure, so the yield stress gradually decreased with the increase of shear rate and shear time in the primary and secondary broken phases. Secondary flocculation increased the yield stress, so the yield stress in the secondary flocculation phase was always higher than that in the primary flocculation phase. However, with the increase of shear action, the gap of the yield stress reduced in the two broken phases.

(2) Shear disruption decreased the average floc size and further decreased the floc strength factors in the broken phases. Secondary flocculation increased the floc strength. However, under high shear disruption, the difference between floc size and floc strength in the primary and secondary broken phases was small.

(3) The floc structure and its fractal dimensions in the four different phases were compared. It is found that the loose primary floc structure was the internal cause of its low shear resistance. Therefore, the sizes of the primarily broken flocs were smaller. Secondary flocculation enhanced the density of the floc structure, and the fractal dimensions were also larger.

(4) Through analyzing the variation of the yield stress, the floc strength factor, and the floc fractal dimension under different shearing conditions, it is found that their variation trend was essentially similar to the increase of shear. In other words, it can be considered that secondary flocculation increased the yield stress of the slurry by enhancing the density of the floc network structure and the floc strength.

(5) The internal reasons for the stronger shear resistance of the secondary flocculating slurry were the increase of the number of binding sites, the electric neutralization between the two flocculants, and the steric hindrance effect of the flocculants.

\section{Data Availability}

All data, models, and code generated or used during the study appear in the submitted article. No additional data are available.

\section{Conflicts of Interest}

The authors declare that they have no conflicts of interest.

\section{Acknowledgments}

The authors would like to thank the China Scholarship Council, the National Natural Science Foundation of China (51834001), the University of British Columbia, and the University of Science and Technology, Beijing.

\section{References}

[1] J. Gregory and S. Barany, "Adsorption and flocculation by polymers and polymer mixtures," Advances in Colloid and Interface Science, vol. 169, no. 1, pp. 1-12, 2011.

[2] C. H. Lee and J. C. Liu, "Sludge dewaterability and floc structure in dual polymer conditioning," Advances in Environmental Research, vol. 5, no. 2, pp. 129-136, 2001.

[3] G. Petzold, M. Mende, K. Lunkwitz, S. Schwarz, and H.-M. Buchhammer, "Higher efficiency in the flocculation of clay suspensions by using combinations of oppositely charged polyelectrolytes," Colloids and Surfaces A: Physicochemical and Engineering Aspects, vol. 218, no. 1-3, pp. 47-57, 2003.

[4] M. G. Vieira and A. E. C. Peres, "Effect of rheology and dispersion degree on the regrinding of an iron ore concentrate," Journal of Materials Research and Technology, vol. 2, no. 4, pp. 332-339, 2013.

[5] Z. Ding, Z. Yin, L. Liu, and Q. Chen, "Effect of grinding parameters on the rheology of pyrite-heptane slurry in a laboratory stirred media mill," Minerals Engineering, vol. 20, no. 7, pp. 701-709, 2007.

[6] J. Yue, Rheological effects on ultra-fine grinding in stirred mills, Ph.D. Dissertation, University of British Columbia, Vancouver, Canada, 2003.

[7] Q. D. Nguyen and D. V. Boger, "Application of rheology to solving tailings disposal problems," International Journal of Mineral Processing, vol. 54, no. 3-4, pp. 217-233, 1998.

[8] E. Burdukova, M. Becker, B. Ndlovu, B. Mokgethi, and D. A. Deglon, "Relationship between slurry rheology and its mineralogical content," in Proceedings of the 24th InternationalMinerals Processing Congress, China Scientific Book Service Co. Ltd., Beijing, China, pp. 2169-2178, September 2008.

[9] D. J. Jeffrey and A. Acrivos, "The rheological properties of suspensions of rigid particles," AIChE Journal, vol. 22, no. 3, pp. 417-432, 1976.

[10] F. Boylu, H. Dinçer, and G. Ateşok, "Effect of coal particle size distribution, volume fraction and rank on the rheology of coal-water slurries," Fuel Processing Technology, vol. 85, no. 4, pp. 241-250, 2004.

[11] E. S. Mosa, M. Abdel-Hady, T. A. T. Saleh, and A. M. ElMolla, "Effect of chemical additives on flow characteristics of 
coal slurries," Physicochemical Problems of Mineral Processing, vol. 42, pp. 107-118, 2008.

[12] R. F. Probstein, M. Z. Sengun, and T. C. Tseng, "Bimodal model of concentrated suspension viscosity for distributed particle sizes," Journal of Rheology, vol. 38, no. 4, pp. 811-829, 1994.

[13] T. R. Camp, D. A. Root, and B. V. Bhoota, "Effects of temperature on rate of floc formation," American Water Works Association, vol. 32, no. 11, pp. 1913-1927, 1940.

[14] T. R. Camp, "Velocity gradients and internal work in fluid motion," Boston Society of Civil Engineers, vol. 30, pp. 219-230, 1943.

[15] C. Ancey and H. Jorrot, "Yield stress for particle suspensions within a clay dispersion," Journal of Rheology, vol. 45, no. 2, pp. 297-319, 2001.

[16] M. Z. Sengun and R. F. Probstein, "Bimodal model of slurry viscosity with application to coal-slurries. Part 1 . Theory and experiment," Rheologica Acta, vol. 28, no. 5, pp. 382-393, 1989.

[17] M. Z. Sengun and R. F. Probstein, "Bimodal model of slurry viscosity with application to coal-slurries. Part 2. High shear limit behavior," Rheologica Acta, vol. 28, no. 5, pp. 394-401, 1989.

[18] P. Ofori, A. V. Nguyen, B. Firth, C. McNally, and O. Ozdemir, "Shear-induced floc structure changes for enhanced dewatering of coal preparation plant tailings," Chemical Engineering Journal, vol. 172, no. 2-3, pp. 914-923, 2011.

[19] M. J. McGuire, J. Addai-Mensah, and K. E. Bremmell, "Improved dewaterability of iron oxide dispersions: the role of polymeric flocculant type, $\mathrm{pH}$ and shear," Asia-Pacific Journal of Chemical Engineering, vol. 3, no. 1, pp. 18-23, 2008.

[20] B. Gladman, R. G. De Kretser, M. Rudman, and P. J. Scales, "Effect of shear on particulate suspension dewatering," Chemical Engineering Research and Design, vol. 83, no. 7, pp. 933-936, 2005.

[21] K. Scales, A. Puisto, J. Paltakari, M. Alava, and T. Maloney, "The influence of shear on the dewatering of high consistency nanofibrillated cellulose furnishes," Cellulose, vol. 20, no. 4, pp. 1853-1864, 2013.

[22] M. S. Nasser and A. E. James, "Effect of polyacrylamide polymers on floc size and rheological behaviour of kaolinite suspensions," Colloids and Surfaces A: Physicochemical and Engineering Aspects, vol. 301, no. 1-3, pp. 311-322, 2007.

[23] A. J. McFarlane, K. E. Bremmell, and J. Addai-Mensah, "Optimising the dewatering behaviour of clay tailings through interfacial chemistry, orthokinetic flocculation and controlled shear," Powder Technology, vol. 160, no. 1, pp. 27-34, 2005.

[24] S. Farrokhpay, "The importance of rheology in mineral flotation: a review," Minerals Engineering, vol. 36-38, pp. 272-278, 2012.

[25] D. S. Parker, W. J. Kaufman, and D. Jenkins, "Floc breakup in turbulent flocculation processes," Journal of the Sanitary Engineering Division, vol. 98, pp. 79-99, 1972.

[26] R. J. François, "Strength of aluminium hydroxide flocs," Water Research, vol. 21, no. 9, pp. 1023-1030, 1987.

[27] P. T. Spicer and S. E. Pratsinis, "Shear-induced flocculation: the evolution of floc structure and the shape of the size distribution at steady state," Water Research, vol. 30, no. 5, pp. 1049-1056, 1996.

[28] J. J. Ducoste and M. M. Clark, "The influence of tank size and impeller geometry on turbulent flocculation: I. Experimental," Environmental Engineering Science, vol. 15, no. 3, pp. 215-224, 1998.
[29] C. Biggs and P. A. Lant, "Activated sludge flocculation: online determination of floc size and the effect of shear," Water Research, vol. 34, no. 9, pp. 2542-2550, 2000.

[30] R. J. Farris, "Prediction of the viscosity of multimodal suspensions from unimodal viscosity data," Transactions of the Society of Rheology, vol. 12, no. 2, pp. 281-301, 1968.

[31] J. A. Mangels and R. M. Williams, "Injection molding ceramics to high green densities," American Ceramic Society Bulletin, vol. 62, pp. 601-606, 1983.

[32] R. E. Johnson and W. H. Morrison, "Ceramic powder dispersion in nonaqueous systems," Advances in Ceramics, vol. 21, p. 323, 1987.

[33] M. A. Yukselen and J. Gregory, "The reversibility of floc breakage," International Journal of Mineral Processing, vol. 73, no. 2-4, pp. 251-259, 2004. 